\title{
EDUCACIÓN
}

\section{Las estrategias de aprendizaje cooperativo utilizadas por los docentes de la Escuela Básica No 23 España, durante el año lectivo 2014}

\author{
Jorge Ayala1', Carlos María Quiñonez Franco
}

\begin{abstract}
Resumen
Introducción: El trabajo cooperativo parte de la premisa de que la cooperación optimiza mejor que la competición el aprendizaje individual de la persona; ello significa que mejora el rendimiento académico de forma individual y grupal en cualquiera de las áreas y edades. Ergo surge la investigación acerca de las Estrategias de Aprendizaje Cooperativo, utilizadas por los docentes con los estudiantes del área de Ciencias Sociales, en el Tercer Ciclo, Turno Mañana de la E.E.B., en la Institución № 23 "España" ciudad de San Lorenzo, durante el año lectivo 2014.
\end{abstract}

Objetivo: Describir el planteamiento que realizan los docentes, para implementar el trabajo cooperativo en el aula.

Método: Enfoque, Cualitativo, la recolección de datos ocurre en ambientes naturales y se describen situaciones de la realidad estudiada, las técnicas de recolección de datos utilizadas: Observación de clases y la entrevista a docentes, instrumentos Guía de Observación de Clase y Cuestionario. Se aplicó una Encuesta a Estudiantes. Cuantitativo, fueron analizadas estadísticamente datos numéricos, que se obtuvieron con la aplicación del cuestionario a los estudiantes, se recolectó información que permitió contrastar la realidad encontrada. Diseño no experimental, sin manipulación de variables, nivel descriptivo, pues buscó caracterizar las Estrategias de Aprendizaje Cooperativo, utilizadas por los docentes en el área de Ciencias Sociales.

Resultados: Los grupos de trabajo cooperativo, se conforman según criterios: el $51 \%$ de los estudiantes ha respondido que es por afinidad de los integrantes; el $20 \%$ de acuerdo al rendimiento escolar; el $13 \%$ al azar; mientras que un $11 \%$ responde que lo conforman de otras formas y un $5 \%$ los estudiantes de mayor rendimiento escolar forman un grupo mientras que los de menor rendimiento forman otro grupo. El 52\% de los encuestados declara, que su grupo de Trabajo Cooperativo se mantiene por un espacio de unas pocas horas de clase; el 38\% que duran todo un año, el $5 \%$ un mes y por último el $5 \%$ un semestre. Las

\footnotetext{
1. Instituto Superior de Educación "Dr. Raúl Peña".

El trabajo corresponde a una Tesis de Grado para la Licenciatura en Ciencias de la Educación.

E-mail: liccmqf@gmail.com
}

DOI: $10.26885 /$ rcei.foro. 2018.58 
técnicas de trabajo cooperativo con la cual trabajan en grupo constituyen, el $53 \%$ de los encuestados responden que es la Investigación en grupo, el 20\% que es el Trabajo por Equipo de Logro Individual (TELI); el 10\% que es la Lectura y Escritura Integrada Cooperativa (LEIC); el 7\% que utilizan otras técnicas, un 6\% responde Torneos de Juegos por Equipos (TJE), un 2\% la técnica de la Enseñanza Acelerada por equipos, y por último el $2 \%$ no contesta, no responde.

Conclusiones: La mayoría de los docentes conocen y aplican los principios básicos en que se sustenta el aprendizaje cooperativo. El tipo de aprendizaje cooperativo establecido en el aula es el Grupo Formal de Aprendizaje Cooperativo. Aquellos conocen acerca de las diversas técnicas de aprendizaje cooperativo que se pueden aplicar en el aula. Los alumnos manejan los conceptos sobre estas técnicas y con la cual generalmente trabajan. Del cruzamiento de datos realizado a través de los diferentes instrumentos aplicados, se determinó que la técnica de aprendizaje cooperativo más utilizada es la Investigación en Grupo. Dependiendo del tipo de trabajo u objetivo estas técnicas pueden ir variando.

Palabras clave: estrategias de aprendizaje cooperativo, técnicas de aprendizaje cooperativo, rendimiento académico.

\section{Referencias}

Aguado, M. J. (2003). Educación Intercultural y Aprendizaje Cooperativo. Madrid: Pirámide.

Barnett, L. E. (2003). Motivación, tratamiento de la diversidad y rendimeinto académico. El aprendizaje cooperativo. Barcelona : GRAO.

Molina Cena, C. E. (2005). El aprendizaje dialógico y cooperativo. Una práctica alternativa para abordar la experiencia educativa en el aula. Buenos Aires: Mgisterio del Río de la Plata.

Stigliano, D. E. (2006). Enseñar y Aprender en grupos cooperativos. Comunidad de diálogo y encuentro. Buenos Aires: Novedades Educativas .

Tamayo y Tamayo, M. (2007). Metodología formal de la investigación científica. México: LIMUSA. 